\title{
L'Internet et le territoire
}

The Internet and the Territory

\section{Éric Guichard}

\section{(2) OpenEdition}

12 Journals

Édition électronique

URL : http://journals.openedition.org/edc/490

DOI : $10.4000 /$ edc.490

ISSN : 2101-0366

Éditeur

Université Lille-3

\section{Édition imprimée}

Date de publication : 1 octobre 2007

Pagination : 83-98

ISBN : 978-2-9514961-9-4

ISSN : $1270-6841$

Référence électronique

Éric Guichard, «L'Internet et le territoire », Études de communication [En ligne], 30 | 2007, mis en ligne le 01 octobre 2009, consulté le 01 mai 2019. URL : http://journals.openedition.org/edc/490; DOI :

10.4000/edc. 490

Ce document a été généré automatiquement le 1 mai 2019.

(c) Tous droits réservés 


\title{
L'Internet et le territoire
}

\author{
The Internet and the Territory
}

Éric Guichard

\section{Introduction}

1 Dès 1998, Henri Desbois évoquait l'idée qu'existent des territoires de l'Internet, ou qu'au moins le concept géographique du territoire puisse appliquer à l'Internet ${ }^{1}$. Et en 2007, son intuition s'avère juste : l'idée que l'Internet soit virtuel, au sens naïf de " coupé du réel ", est en passe d'être abandonnée, et les tensions, conflits, relatifs à l'Internet, sont manifestes, qu'ils touchent à des formes concrètes du territoire (câbles, juridictions nationales) ou à des formes moins repérables (réseaux sociaux en construction...).

2 Pour autant, il semble difficile d'imaginer que l'Internet soit le catalyseur direct de ce renouveau d'intérêt pour le territoire, sauf à reprendre une forme incantatoire assez commune, qui donne à penser que la technique est magique et que les pratiques sociales qui s'organisent autour d'elle sont spontanées: je vois là une idéologie, ou plutôt une somme d'idéologies ${ }^{2}$ qui tend à nier que les relations entre collectifs soient structurées par des rapports de force.

3 Il me semble que si on prend un point de vue un peu décalé, qui n'aborde pas d'emblée les territoires de l'Internet mais qui explicite la façon dont l'informatique et l'Internet réorganisent, avec la carte électronique, des territoires qui n'ont rien à voir avec des données qui leur soient associées, on pourrait déjà comprendre certaines dynamiques de la territorialisation, et avoir le désir de poser les questions suivantes : quels ingrédients intellectuels facilitent le raisonnement en termes de territoires? Quelles sont les classes sociales ou intellectuelles les plus réceptives à ce concept ? Cette capacité leur donne-telle un surcroît de pouvoir pour forger ces territoires?

4 Si mon raisonnement est juste, l'irruption des territoires de l'Internet ne serait plus la conséquence d'un artifice du type deus ex machina, mais conséquence à la fois des formes contemporaines de l'écriture et du pouvoir des classes et groupes sociaux qui la maîtrisent le plus. Alors, l'Internet ne serait plus ce nouveau monde peuplé de métaphores 
spatiales, où les imaginaires et les territoires s'imposeraient comme de nouvelles évidences témoins du caractère novateur de la «chose» (Mathias, 2002), mais la production prévisible d'une catégorie sociale aujourd'hui dominante - les informaticiens - qui sur-sollicitent, dans leur appréhension du monde, les notions d'espace, et plus implicitement, de territoire. Il sera alors logique que le monde communicationnel qu'ils configurent soit " prêt » à se territorialiser. D'autant que les premiers à l'investir risquent fort d'être ceux qui le conçoivent ${ }^{3}$.

territoire est, pour moi, la «superposition d'un espace localisé et de pratiques sociales » (Guichard, 2006). Point de territoire sans collectif, réel ou rêvé : la carte géologique représente tout sauf un territoire. Plus qu'un espace "réel», le territoire relève de la représentation. Cependant, cette dimension humaine du territoire, sociologique et culturelle, est parfois euphémisée chez les personnes mal à l'aise avec les concepts des sciences humaines. Il semble que cette difficulté à préciser les relations entre culture et territoire explique la préférence pour la notion d'espace. A priori, l'espace est géographique (l'Alsace-Lorraine, l'atelier de métallurgie de l'usine, Los Angeles), mais il peut aussi renvoyer à des objets plus proches d'une construction mentale que d'une géométrie concrète (la Pologne au XIX ${ }^{e}$ siècle). L'espace est donc une notion plus neutre, plus plastique que le territoire. On arrive alors à parler d'espace-temps, d'espaces intersidéraux, de cyber-espace... Ceci dit, un espace doté d'enjeux, de conflits, articulé avec des représentations, des pratiques sociales, n'est plus un espace, mais un territoire.

6 Et cette notion arrive vite, à partir de questions simples comme " où-suis-je ? ", « et avec qui ?", qui correspondent à un besoin d'image d'(au moins) un espace vécu, de représentation au sens premier du terme. La somme de cette représentation et de celles qui s'exercent sur elle, c'est le territoire. Cet ensemble itératif de représentations va se développer à l'aide d'une technique intellectuelle.

Évidemment, la technique, l'écriture, va agir sur la représentation. Intrinsèquement, presqu'indépendamment de l'objet représenté, par le biais des contraintes qu'elle impose et des capacités qu'elle offre. Mais la représentation, ou le besoin de représentation, agit à son tour sur la technique : il invite à la raffiner, même si cela prend du temps.

Et le collectif, le social? Il reste bien présent: une représentation est rarement individuelle ou singulière ${ }^{4}$, elle se structure par des échanges, des controverses, des synthèses avec d'autres représentations. Elle est dépendante de références collectives qui ont une histoire et des temporalités : la culture. De même pour la technique : qu'elle soit intellectuelle ou pas, elle est inscrite dans du collectif, même si elle peut s'accorder avec la singularité d'un/e artiste ou d'un/e savant/e. Malinowski va jusqu'à poser l'équation technique $=$ culture (Malinowski, 1968).

\section{Fabriques du territoire}

9 Je voudrais donc montrer ici comment l'électronification-réticulation de l'écriture peut participer de la (re)définition des territoires, indépendamment des réseaux sociaux de l'Internet et des variables propres à l'Internet. Ou comment l'informatique permet des reconfigurations territoriales sans lien avec les territoires de l'Internet, mais qui à mon avis mettent en évidence des ingrédients essentiels à l'essor de ces derniers. fruit d'une écriture popularisée depuis peu: la carte des communes de France, où 
apparaissent progressivement les communes de plus de $10000,9900 \ldots 100$ et 0 habitants (chaque commune est représentée par un point de diamètre constant). Cet exemple, disponible à l'URL: http://barthes.enssib.fr/atelier/geo/communesfr.html, fait apparaître le formidable pouvoir politique des communes rurales en France ${ }^{5}$, et donne à voir une France complètement euphémisée, voire niée : avec un tel outil d'écriture, on montre un territoire réel qui n'a pas sa place dans nos représentations territoriales. L'écriture électronique, l'animation sont-elles déterminantes? A priori non, car trois cartes imprimées, l'une des communes de 10000 habitants et plus, l'autre de ceux de 500 habitants et plus, et la troisième, des 36000 communes, auraient été presque aussi parlantes. Oui, car je crois que tant la chaîne de production de ces cartes de 36000 points, que l'idée de l'apport heuristique de cette réalisation n'était pas pensables en 1950.

Prenons ensuite un objet historiographique désormais courant, l'immigration en France. L'acception contemporaine de ce terme date de la fin du XIX ${ }^{e}$ siècle ${ }^{6}$. Elle fut suffisamment institutionnalisée entre les deux guerres pour mener à la production de recensements, et donc de cartes. Peu importe qu'hier comme aujourd'hui, les définitions de l'objet soient floues, voire incohérentes, et que les espaces dans lesquels on le mesurait le soient tout autant - par exemple, les départements, qui pouvaient aussi bien contenir de vastes zones rurales, sans étrangers, que des foyers industriels qui appelaient et concentraient de nombreux immigrés (Noiriel, 1984).

Un atlas imprimé actuel de cette période obligerait à une édition sélective ${ }^{7}$ : il reprendrait sûrement la majorité des cartes d'antan même si elles seraient aujourd'hui plus nettes et en couleur. Tandis qu'avec l'informatique, en tirant parti de la combinatoire appliquée aux données d'antan (nationalités ${ }^{8}$, taux de féminité, dates des recensements, différentiel entre ces années), on peut multiplier le nombre de cartes: l'atlas de l'immigration: http://barthes.enssib.fr/atlasclio permet d'en réaliser 4000. Ici encore, l'écriture intervient pleinement: les possibilités de manipulation textuelle, de calcul et de production graphique de l'informatique permettent des représentations territoriales inédites; la masse des cartes nourrit la comparaison, qui amplifie et approndondit la réflexion sur la notion de territoire.

Figure 1 : Carte des Italiens en France entre 1931 et 1936. 


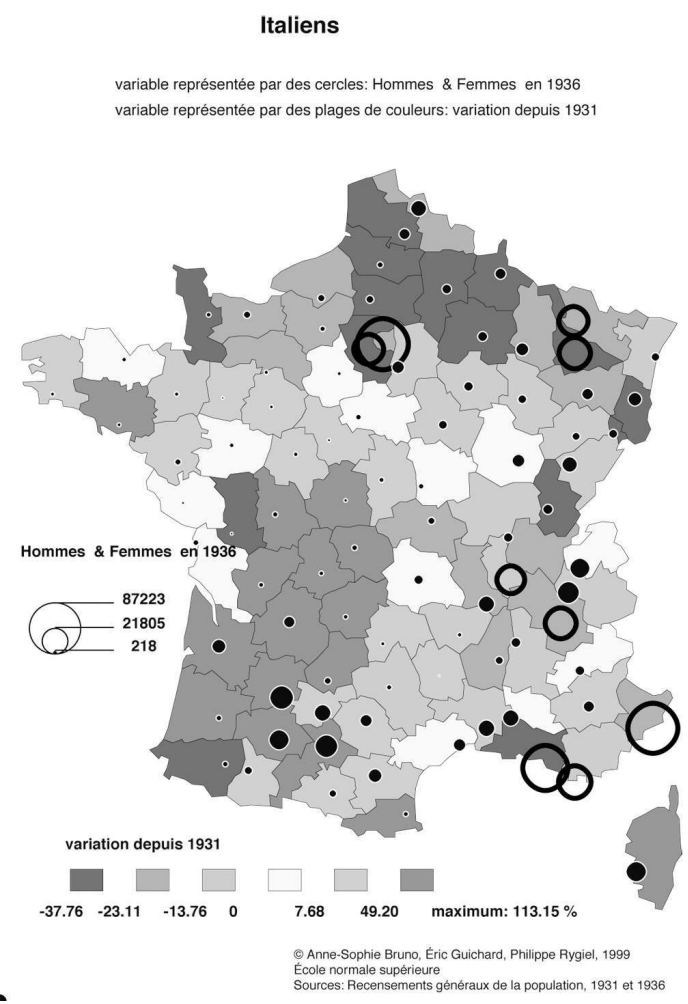

13 On remarque un phénomène désormais bien connu, impensé entre les deux guerres: malgré les nombreuses expulsions d'étrangers entre 1931 et 1936, visibles dans les départements industrialisés (en bleu), des Italiens viennent, de ces départements, mais surtout d'Italie, s'établir dans les zone rurales du Sud-Ouest de la France pour entretenir et moderniser l'agriculture : http://barthes.enssib.fr/atlasclio.

Un tel atlas fait apparaître l'autre composante de la territorialisation: le territoire, appliqué à un thème donné, dépend grandement des personnes qui le sollicitent. Apparaissent de nouvelles configurations territoriales, du fait de l'apparition de nouveaux collectifs : les descendants d'immigrés italiens (cf. carte 1), ou algériens, les passionnés de maquettes futuristes à travers le monde, les universitaires européens, les informaticiens.

J'espère ici avoir mis en évidence deux paramètres, potentiellement indépendants de l'Internet en son sens commun, qui transforment les territoires, les réactualisent, les font émerger :

- Une évolution du système d'écriture qui permet de nouveaux jeux combinatoires (travail sur les listes), calculatoires et graphiques, et la dynamique réflexive qui va avec.

- L'apparition de nouveaux acteurs, qui vont participer à l'écriture du territoire avec les moyens dont ils disposent.

\section{L'écriture}

Le pouvoir de l'écrit sur le territoire pourrait se décomposer en deux facteurs: le premier, propre à l'écriture, relève de l'histoire longue, et agit sur l'ensemble des représentations ; le second est lié à la modernité de cette technologie de l'intellect, même s'il date de bien avant l'invention de l'Internet. Jack Goody (1994), David Olson (1998) et Christian Jacob (2007) ont rappelé les formidables contraintes et capacités qui découlent 
de l'écriture, système de communication s'il en est, aujourd'hui omniprésente pour tous les humains sans exception.

Déjà, si la carte n'est pas l'unique mode d'objectivation du territoire, il est difficile, dans nos sociétés plongées dans l'écriture, d'évoquer la notion de territoire sans l'écrit: le caractère éminemment social du territoire fait qu'il est avant tout construit par une succession de réflexions écrites, d'incantations (politiques : nationalistes, religieuses...) écrites, d'appels à une mémoire écrite. Des commentaires, des critiques, des écoles de pensée, des découvertes, des doxa co-fabriquent le territoire, comme la majorité de nos représentations contemporaines ${ }^{9}$. Et ces représentations sociales, ces «ensembles de conceptions collectives sur le monde et la façon dont il est organisé », sollicitent des outils qui sont autant graphiques que conceptuels pour être précisés, remodelés, transmis, voire créés. Or l'élaboration de cet outillage ne s'est pas faite sans coût : pour que l'écriture et la lecture s'émancipent, entre le XII ${ }^{e}$ siècle et le XVII ${ }^{e}$ siècle, d'une pure subjectivité où la valeur d'illocution était faiblement prise en compte, il a fallu d'une part préciser dans les textes ce qui relevait du fait et ce qui relevait de l'interprétation; d'autre part, compléter le système de signes de l'écriture et l'attacher à des notions précises; et réciproquement : inventer des signes qui expriment de nouvelles notions. D'où l'essor des symboles mathématiques, chimiques - qui sont sûrement tout sauf des symboles et tout sauf des conventions; puis des formes géométriques élémentaires qui favorisaient l'usage des schémas et graphiques, qui entraient dans le domaine de la preuve visuelle: segments, flèches, polygones, courbes, qui, dotés d'une syntaxe, permettaient de réaliser figures, graphiques, etc.

Cette tendance est très favorable au développement de la notion de territoire, qui fait appel à une logique très visuelle, et invite à une appréhension spécifique, héritée de son rapport au spatial. Tout d'abord, le territoire n'a qu'une faible connotation positive : tout être sait qu'il parcourt, physiquement, socialement, culturellement, divers territoires ; le seul qui n'en ait éventuellement qu'un, c'est l'autre. Ainsi, la construction mentale du territoire passe par une somme de représentations (au sens premier du terme), d'images de photographies en négatif, pourrait-on dire - qui se traduisent en une résultante assez floue et abstraite, qui finit par produire des contours, des frontières, des pôles, des gradients. Autant de notions qui sont certes produites par nos cultures de l'écrit, mais qui peuvent difficilement s'exprimer sans une écriture spécifique, fortement teintée de géométrie, quasi topologique. Ainsi, le territoire contemporain est-il le produit d'un charroiement intellectuel qui combine de l'émotion, des formes de perception intuitives ou élaborées, singulières et collectives, des faits, des synthèses, et un outillage lourd de figures complexes, héritées de la physique et de la mathématique qui, elles-mêmes, ont puisé dans ces problématiques de la réalité, de la nature et de la représentation pour se développer, s'autonomiser, et inventer les outils d'écriture ad hoc.

Cette transformation de l'écriture, son absorption de la géométrie, a pris du temps : au début du XIX siècle, les mathématiciens rédigent encore leurs textes de façon fort « littéraire ». Peu de formules, peu de graphiques. Ce n'est qu'au milieu de ce siècle que les unes et les autres commencent à se répandre. De façon tout à fait prévisible, cet usage d'une écriture généralisée se diffuse très vite dans toutes les couches de la société, pour étendre les possibilités du raisonnement, de la preuve, de la (dé)monstration : Larousse popularise les graphiques, formules, planches et partitions de musique dans son dictionnaire universel. Les cartes thématiques se multiplient, plus sous l'impulsion des 
ingénieurs que des géographes (Palsky, 1996). Chiffres, formules, graphiques vont structurer la science sociale naissante ${ }^{10}$.

Lentement, mais sûrement, les objets qui, a priori relevaient plus de l'image (photographie, peinture...) que du texte, plus du planaire et du spatial que du du linéaire, se sont trouvés rabattus vers le texte. Au point que des couches complexes ont été inventées et affinées dans le domaine de l'informatique pour que tout ce qui relève du graphique organisé, et plus précisément de la géométrie (élémentaire) relève maintenant (et depuis environ 30 ans) du texte. Par exemple, une flèche s'écrit : \$\rightarrow\$ (en LateX) et un cercle, aux paramètres aisément lisibles s'écrit <circle $\mathrm{cX}=\ll 1550$ » $\mathrm{cy}=\ll 900$ » $r=« 20 » />$ (en SVG). Les bureaux de nos ordinateurs, avec leurs icônes polygonales, ne sont-ils pas la preuve de cette géométrisation de l'écriture?

21 L'intrusion du graphique dans le système sémiologique propre à l'écriture date donc d'un siècle et demie. Mais ce second facteur (le récent) est très lié au premier : tout comme on peut relier la construction de la science au XVII ${ }^{e}$ siècle aux prémisses de la méthodologie de l'interprétation du XII ${ }^{e}$ siècle, puis le plein essor de la pensée scientifique au XIX ${ }^{\mathrm{e}}$ siècle à ce début de la période moderne, il est aisé de relier l'écriture actuelle à ses transformations du XIX siècle. Il y a 150 ans, les scientifiques ont eu besoin d'une écriture qui intègre des graphies étranges et des éléments de géométrie, en même temps qu'ils en ont compris les avantages. Parce qu'ils ont perçu la profonde interdépendance de la pensée et de l'écriture, de la pensée et de la technique, ils ont mécanisé l'écriture, avec les utopies, les échecs et les succès que l'on connaît. C'est suite à ce mouvement intellectuel, inscrit dans la durée comme dans le collectif, que l'informatique contemporaine « peut » traduire, transmettre, organiser tant de signes, que la géométrie s'est intégralement textualisée. Bien plus qu'une technique réservée à des militaires, l'informatique semble être la science de la technique de l'intellect, la techno-logie. Et l'écriture qu'elle permet est un paramètre essentiel de la construction des territoires actuels.

Figure 2: Carte topologique des AS de l'Internet ${ }^{11}$. 


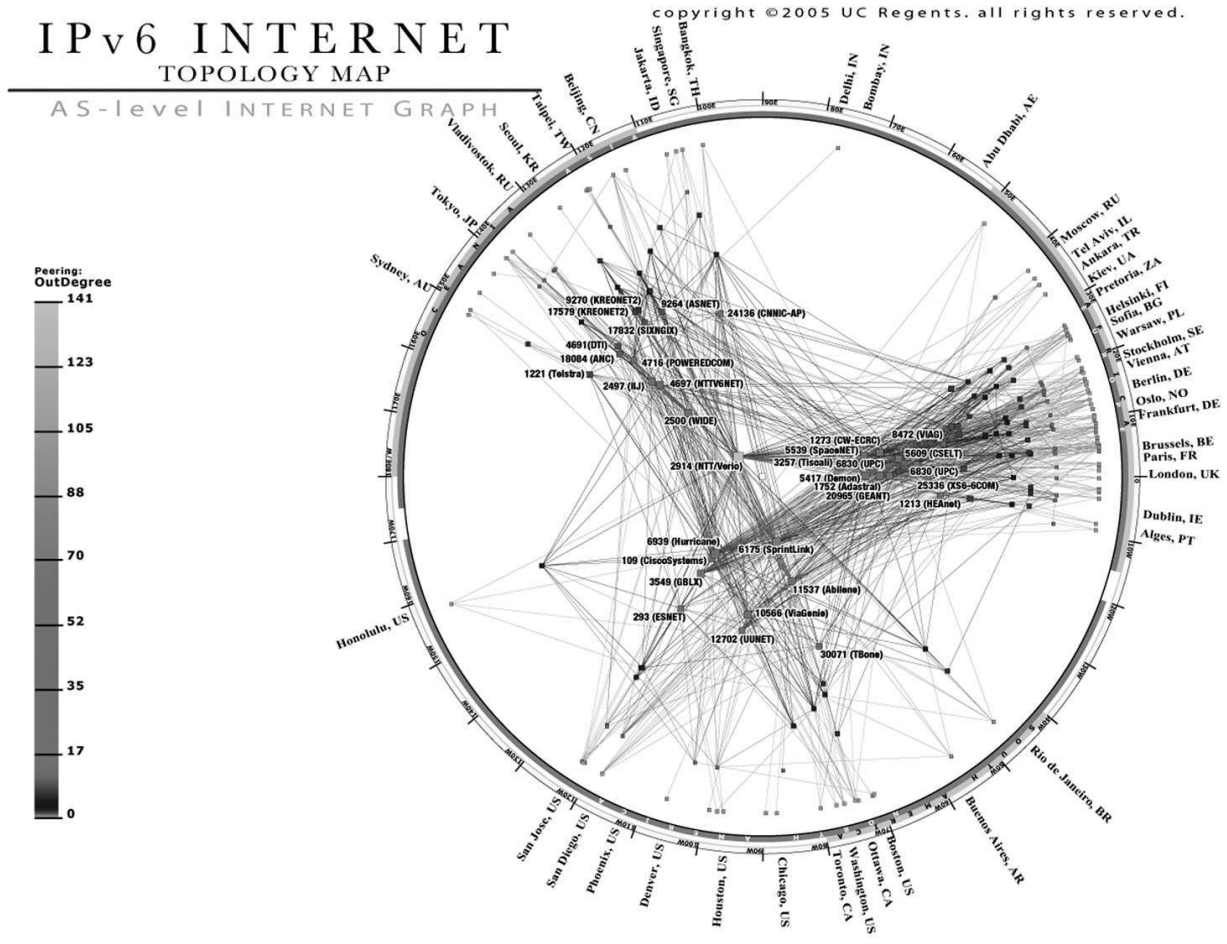

\section{Territoires de l'Internet}

De nombreuses cartes de l'Internet, c'est-à-dire des représentations graphiques plus ou moins reliées au monde terrestre et issues de données propres à l'Internet (flux Internet, réseaux d'échanges électroniques...), ont été produites ces dernières années. Et leur diffusion s'est faite très rapidement. Le site : http://www.cybergeography.org/ est assez révélateur de cette profusion, au moins jusqu'en 2004, date à laquelle il a cessé d'être alimenté.

Cette carte, du groupe de recherche CAIDA $^{12}$, est un bon exemple de ce genre graphique qui a fait le tour du monde. Ses auteurs avaient le projet de décrire la quasi-totalité des flux Internet, à partir des données récupérées auprès de leurs propriétaires (fournisseurs de câbles ou gestionnaires de grands réseaux). Même si nous avons du mal à lire le graphe multicolore du milieu, nous voyons qu'il est inscrit dans un cercle qui pourrait représenter l'écorce terrestre. En tout cas, des noms de villes sont clairement affichés. Avec une telle carte, nos représentations territoriales sont assez malmenées. Certes, nous avons compris que la géodésie n'est pas la métrique choisie par nos chercheurs. Il n'empêche que cette carte positionne Paris plus loin d'Helsinki et de Moscou que Pretoria (cf. détail figure 3). Ensuite, des villes comme San Jose, San Diego et Phoenix ont autant d'importance que les capitales européennes (cf. figure 4).

Figure 3 : Détail de la carte 2 « distances » entre Pretoria, Paris et Helsinki. 


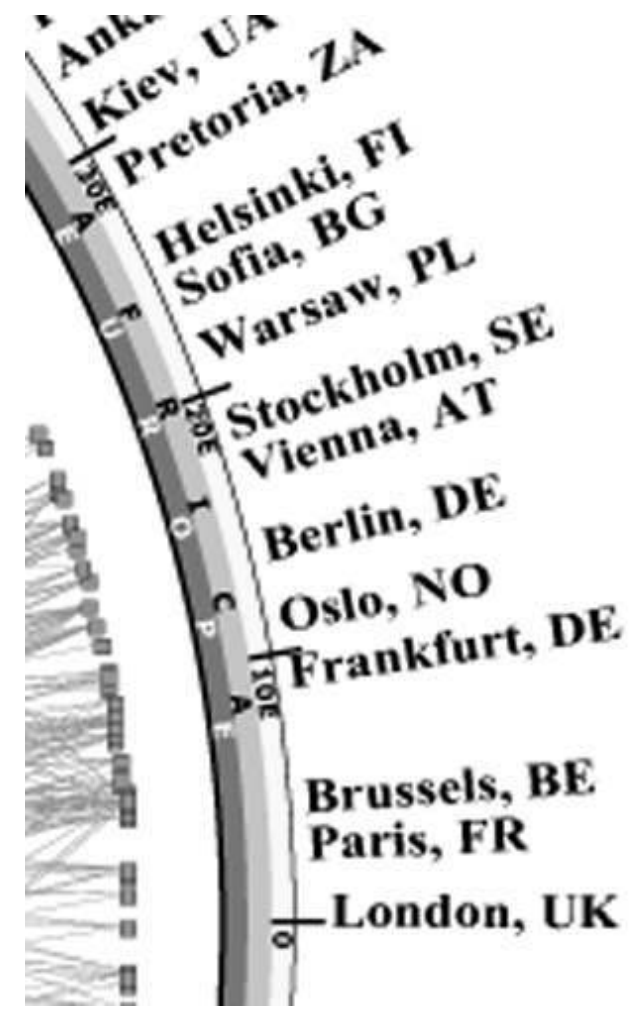

Figure 4 : Détail de la figure 2 : importance des villes californiennes.

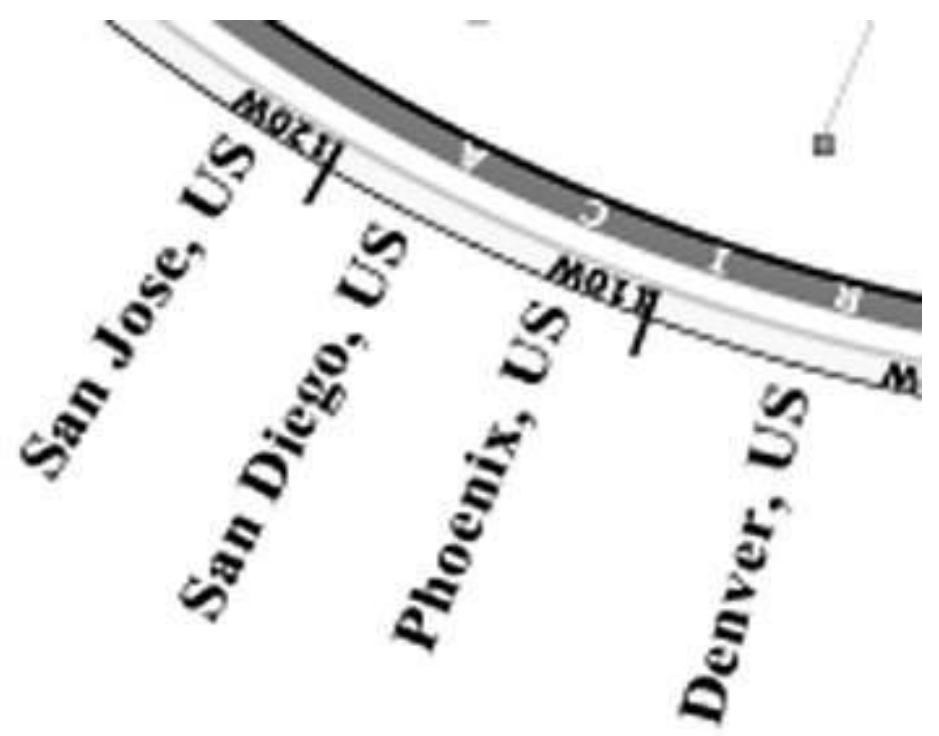

Avec cet exemple, un groupe qui a une claire compétence en matière de littératie (récupération de données, traitement, mise en forme graphique...) construit le territoire comme il l'entend: classiquement, on a une carte de propriétaires, et les villes sont repoussées à sa périphérie en même temps qu'elles sont l'unique renvoi au réel : point de fleuve, de montagne, de campagne, de frontière, ou même d'État. Ces absences nous aident à percevoir la force idéologique d'une telle carte, même si elle est implicite.

Il me semble que le territoire de l'Internet est avant tout le résultat de cette écriture cartographique collective d'un groupe aux motivations précises, les métrologues de 
l'Internet, et aux compétences étendues en matière d'écriture contemporaine, géométrisée. En ce sens, ils sont les descendants des ingénieurs du XIX ${ }^{\mathrm{e}}$ siècle qui ont inventé les cartes « spéciales », que l'on appelle aujourd'hui « thématiques ».

On repère dans la foule de cartes de l'Internet une « certaine similitude esthétique ", avec l'emprunt de " codes visuels issus des représentations graphiques de la science-fiction " (Desbois, 2007). Il y aurait effectivement lieu de comprendre en quoi et comment ces cartes participent d'une ré-écriture du monde, en même temps qu'elles sont le produit d'une culture précise. De même, il faudrait étudier en détail comment ces territoires écrits, créés, sont vécus par leurs auteurs ; et comment, au-delà de la force culturelle dont ils héritent, ces territoires deviennent des matrices de territoire prêtes à être investies par des groupes sociaux, des imaginaires. Comment, de potentiels, d'ancrages de l'imaginaire, ces territoires se transforment en creusets de territoires vécus, de cultures, et bien sûr, de conflits. C'est sur ces bases que se constitue une géographie de l'Internet, et que la problématique du territoire s'avère précieuse.

Je voudrais revenir sur les capacités que nous offre l'écriture électronique et réticulée. Nous avons vu que cette écriture moderne a eu le temps d'être socialisée. Au point qu'il me semble qu'aujourd'hui, la notion de littératie, qui signifie l'outillage mental nécessaire pour maîtriser la technologie de l'intellect qu'est l'écriture, ne se résume pas à l'art de savoir fouiller dans une encyclopédie imprimée et dans un moteur de recherche, mais s'étend plus généralement à celui de savoir combiner et lire ce système de signes étendus composé à la fois de l'alphabet et de ces formes graphiques ${ }^{13}$. Et il y a de grandes chances pour que les lettrés d'aujourd'hui soient, à l'image de ceux d'hier, celles et ceux qui maitrisent au mieux la technologie de l'intellect du moment, avec tout ce que cela signifie : savoir lire ces textes, hypertextes, graphiques, listes ; savoir écrire avec ${ }^{14}$; avoir conscience des avantages et limites de cette écriture et des précédentes (interprétation, valeur d'illocution de leurs propres textes ou de ceux d'autrui); comprendre les dynamiques intellectuelles et sociales du moment et du passé ; comprendre la réflexivité de l'écriture.

De plus en plus, ces lettrés sont informaticiens. Cela ne signifie pas pour autant que les seuls héritiers des érudits se recrutent chez les titulaires d'une thèse d'informatique: j'imagine que dans cette discipline, la proportion de personnes ayant un recul réflexif sur leur pratique d'écriture est sensiblement la même que dans les autres. Mais nous ne devons pas oublier le nombre croissant de non-informaticiens qui ont acquis d'étonnantes compétences en matière d'écriture électronique à cause des problématiques propres à leur discipline : linguistes, antiquistes, historiens, etc.

29 Aujourd'hui comme hier, ces lettrés sont juges et partie : juges, car ils analysent, évaluent les représentations sociales; ils précisent leurs structures, leurs tendances, leurs infléchissements. Par exemple, ils expliquent comment se constituent les territoires. Parties, parce qu'ils expriment, dessinent ces représentations, ces territoires. Ils les contraignent donc, du fait qu'ils les inscrivent dans un mode textuel étendu: ils en définissent les possibles.

La nature du territoire, sa perméabilité à la spatialité, en même temps que la capacité actuelle de l'écriture à traiter le graphique et la géométrie, facilitent les jeux d'inscription territoriale, qu'ils reprennent ou non les anciens motifs ${ }^{15}$. En ce sens, l'Internet, vu comme aboutissement actuel de notre technologie de l'intellect, modifie nos perceptions territoriales. Et, quand les experts actuels de l'écriture utilisent cette dernière pour leurs propres besoins - mesurer, donner à voir cette écriture en flux, paquets, électrons - ils 
développent une cartographie spécifique qui invite à voir l'Internet comme un territoire. Il ne manque plus à ce territoire que des "gens " pour l'investir. Certes, ces gens seront les internautes, et nous savons désormais combien l'Internet, avec ses variantes web, mail, listes de discussion, etc. est socialisé, approprié, remodelé. Tout cela est affaire de culture, d'écriture. Mais il me semble illusoire de négliger l'empreinte des auteurs de la matrice. Tout comme il est illusoire d'oublier les nouveaux propriétaires des territoires de l'Internet : à côté d'un Murdoch aux rênes de Myspace, quelle liberté a l'internaute si ce n'est celle de circuler en des chemins bien balisés, en se transformant en hommesandwich aussi souvent que le premier l'exige?

\section{BIBLIOGRAPHIE}

Desbois, H., (2001), « Les territoires de l'Internet : suggestions pour une cybergeographie », in : Éric Guichard (dir.), Comprendre les usages de l'Internet, pp. 253-263, Éditions Rue d'Ulm, Paris, 2001.

Desbois, H., (2006), « L'Internet et la mondialisation », in : Isabelle Lefort et Vincent Moriniaux (dir.), La mondialisation, du Temps (éd.), Nantes, 2006.

Desbois, H., (2007), « Présence du futur, le cyberespace et les imaginaires urbains de sciencefiction », Gographie et cultures, $61: 121-138,2007$.

Goody, J., (1994), Entre l'oralité et l'écriture, Presses Universitaires de France, Paris, 1994.

Guichard, É., (2004), « L'Internet, une technique intellectuelle », in : Éric Guichard (dir.), Mesures de l'Internet, pp. 19-49, Les Canadiens en Europe (éd.), Paris, 2004.

Guichard, É., (2006), « L'Internet : retrouvailles de l'écriture et de la cartographie », Revue de la Bibliothèque nationale de France, 24 : 51-55, 2006.

Jacob, C., (dir.), (2007), Lieux de Savoir. Espaces et communautés, Albin Michel, Paris, 2007, Volume I. Malinowski, B., (1968), Une théorie scientifique de la culture, Points, François Maspero, Paris, 1968.

Mathias, P., (2002), « La chose Internet », in : Éric Guichard et Jacques Lajoie (dir.), Odyssée Internet : enjeux sociaux, pp. 41-59, Presses de l'Université du Québec, 2002.

Noiriel, G., (1984), Longwy. Immigrés et prolétaires, 1880-1980, P.U.F., Paris, 1984.

Olson, D., (1998), L'univers de l'écrit, Retz, Paris, 1998.

Palsky, G., (1996), Des chiffres et des cartes. La cartographie quantitative au XIX ${ }^{e}$ siècle, CTHS, Paris, 1996.

\section{NOTES}

1. Communications à l'Atelier Internet de l'ENS et au colloque Comprendre les usages de l'Internet, ENS, 1999. Pour une version écrite : (Desbois, 2001). Voir aussi (Desbois, 2006). 
2. Nous sommes nombreux à penser que l'étude des liens entre idéologies et mythologies (Guichard, 2004) nourrit, à partir de faits concrets, une anthropologie des mondes contemporains en même temps qu'une épistémologie.

3. Je crois assez peu à l'idée d'un détournement massif et spectaculaire de l'Internet par ses utilisateurs. Un tel discours tend à signifier que l'Internet serait enfin devenu une technique neutre, banale, absorbée par notre culture. Vœu pieux des petits et grands prêtres de l'Internet, qui voudraient qu'avec l'Internet, les lentes temporalités nécessaires à l'appropriation d'une technique - même intellectuelle - par les sociétés se réduisent à une dizaine d'années, et qui, une fois de plus, valorisent, pour le plus grand bénéfice de ses propriétaires, un Internet inodore et indolore, voire centré sur le plaisir. Ceci dit, la relation de l'Internet à l'écriture fait que l'on peut constater une variété de pratiques, prévues ou inattendus, du fait du caractère intellectuel de ces pratiques, et du fait qu'avant elles laissaient beaucoup moins de traces aisément utilisables.

4. Et si elle l'est, c'est durant un temps bref.

5. La moitié des communes françaises ont moins de 500 habitants. Il s'ensuit, par le jeu des élections aux conseils départementaux, régionaux et national (Sénat), qu'un électeur rural vaut 200 électeurs parisiens. Date des données : 1992.

6. Au début de ce XIX ${ }^{\mathrm{e}}$ siècle (Restauration), les immigrés étaient les nobles émigrés qui rentraient en France. Source : Gérard Noiriel, communication personnelle.

7. Du fait de contraintes diverses: coûts de production, poids maximal de l'ouvrage, public potentiel, etc.

8. Définies comme telles à l'époque, opérant un singulier mélange des genres entre nationaux d'un pays précis, sujets français des colonies qui n'ont pas la nationalité française, et plusieurs rubriques « autres »...

9. Et il n'y a aucune difficulté à intégrer dans l'écriture des formes de communication qui semblent oralisées : un journal télévisé est clairement écrit.

10. Cette évolution peut s'interpréter comme un progrès. Mais il serait déraisonnable de penser que ses conséquences relèvent aussi du progrès : cf. l'usage bien peu rationnel que les eugénistes des années trente ont fait de la mathématisation de la sociologie.

11. AS : collection de routeurs dont la politique d'acheminement unique est coordonnée par un exploitant de réseau (propriétaire). Copyright 2005 The Regents of the University of California All Rights Reserved : http://www.caida.org.

12. Cooperative Association for Internet Data Analysis, centre de recherche en métrologie de l'Internet, San Diego : http://www.caida.org/.

13. Le fait n'est pas nouveau: avant l'extension de ce système graphique, la littératie ne se réduisait pas à la compréhension des agencements possibles d'une trentaine ou d'une soixantaine de signes (si l'on distingue majuscules des minuscules). Par exemple, dès les débuts de l'écriture, se posait la question de leur contexte, intellectuel, social, culturel.

14. Dans ce contexte, savoir lire et écrire requiert aussi de savoir «manipuler» des logiciels. Mais ces derniers relevant aussi de l'écriture (ce ne sont que du texte), il me semble inutile d'insister sur leur spécificité, à mon avis abusivement évoquée quant on évoque la notion de littératie électronique.

15. Par exemple, de nombreux auteurs de logiciels de cartographie font fi de la sémiologie graphique développée par Bertin. 


\section{RÉSUMÉS}

Depuis une dizaine d'années émerge l'idée que l'Internet est susceptible de créer des territoires. Pour aborder cette question, je commencerai par étudier la façon dont des territoires « classiques » peuvent être reconfigurés par l'Internet. Cela devrait préciser le rôle de l'écriture dans la fabrication et la transformation de ces territoires. Ensuite, j'aborderai les territoires numériques et leurs auteurs.

For about ten years, more and more scientists have come to see the Internet as a source of territories. To deal with this issue, this paper studies how traditional territories can be reshaped by the Internet. This should specify the rules of writing in the construction and transformation of territories. The paper then addresses digital territories and their authors.

\section{INDEX}

Mots-clés : technologie de l'intellect, internet, territoire

Keywords : technology of the intellect, internet, territory

\section{AUTEUR}

\section{ÉRIC GUICHARD}

Éric Guichard est maître de conférences à l'École nationale supérieure des sciences de l'information et des bibliothèques (Enssib), Responsable de l'équipe Réseaux, Savoirs \& Territoires de l'École normale supérieure (ENS, Paris). Ses domaines de recherche sont : usages de l'Internet, géographie de l'Internet, écriture, mondes savants et lettrés, pratiques intellectuelles, édition savante électronique. Productions en lien avec ces recherches : cartographie de l'Internet, et sur l'Internet (atlas en ligne). Adresse électronique :Eric.Guichard@enssib.fr. 